\title{
TERRESTRIAL MOBILE MAPPING: PHOTOGRAMMETRIC SIMULATOR
}

\author{
C. Taglioretti, A. M. Manzino
}

DIATI Department, Politecnico di Torino, Corso Duca degli Abruzzi 24, 10129 Torino, Italy - (cinzia.taglioretti, ambrogio.manzino)@polito.it

KEY WORDS: Path accuracies, Simulation, Mobile Mapping, Planning trajectory, Terrestrial navigation

\begin{abstract}
:
Nowadays many types of sensors are used for terrestrial mobile mapping (TMM): IMU, odometers, GNSS, cameras, etc., and it is essential to understand how these sensors can improve the solution in terms of precision, accuracy and reliability. TMM issues are characterized by many variables: vehicle trajectory, the height of the buildings and the distance between them, traffic conditions, the presence or absence of trees, the level of illumination, etc.

The aim of this study is to determine how photogrammetric measurements can improve the quality of TMM solution at least concerning magnitude and error propagation when there is no GNSS signal (for example in an urban canyon). Another purpose of the study was to determine the most suitable design project for a specific relief in order to obtain the best possible photogrammetric results.

By analyzing the error propagation in the various components of relative orientation along the trajectory and considering a sequence of images characterized by an overlap varying between 60 to $90 \%$ and the same number of tie points, results were obtained which confirmed the reliability of the data produced by the simulator. These results are shown in this paper.
\end{abstract}

\section{INTRODUCTION}

When we want to investigate the precision obtainable with photogrammetry in a Terrestrial Mobile Mapping (De Agostino, 2009), we do not generally have a database containing enough varied photogrammetric data to be able to investigate the whole spectrum of case studies of TMM reliefs (El-Sheimy, 1996). And it is unthinkable, both from an economic point of view and from the point of view of "time" (Hassan et al, 2007), that anyone can adopt many different databases created acquiring personally data in the field.

In order to solve this problem and have various types of datasets at disposal, the Authors created a simulator of "sequence of images" with Matlab®, which enabled them to change the inputs and therefore produce the type of relief required.

From a photogrammetric point of view, the totality of TMM reliefs varies thanks to the parameters, which are the inputs of the simulator (shown in Figure 1). These parameters refer to the camera used for the relief, the type of frames, the vehicle trajectory and the terrain point distribution.

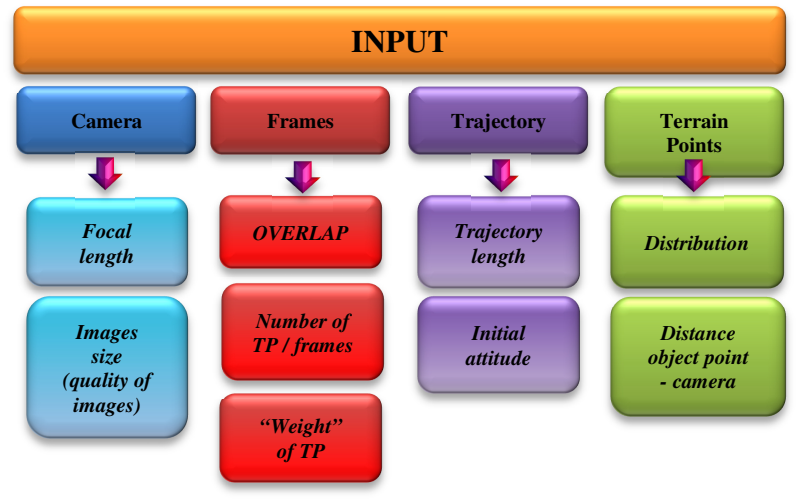

Figure 1. Input parameters

\section{DESCRIPTION OF THE INPUTS}

As already mentioned in the introduction, the number of the indispensable parameters is required for realizing a photogrammetric relief and it is essential to have a good understanding of them for achieving a successful TMM relief and more importantly they can be set as input.

The type of camera is the first parameter that influences the quality of the frames. An expensive camera can be used or a low cost camera with a reduced focal length according to the current trends of research.

The Authors opted for the latter, in fact they simulated using of a low cost camera Lynx Optech, by setting its characteristics (focal length, image resolution and field of view) as inputs of the program. The simulated results, which can be seen in the next section of this paper, are comparable with the results obtained by analyzing a real case study carried out with the same camera and share the same characteristics in terms of type of trajectory and distance camera-terrain points.

The dimension of the frames is an important factor affecting quality and is another parameter of the simulator. This parameter is related to the quality of the camera and image resolution is generally lower when a cheap camera is used.

Since the aim of this study is to determine how photogrammetric measurements can improve the quality of TMM solution when GNSS signal is absent, an important issue is the overlap between two successive frames.

The overlap can range between a minimum value of $60 \%$ to a maximum value of $90 \%$ and it is essential for determining the relative orientation between the couple of successive frames, and affects the quality of the orientation results.

Another important aspect is to establish how many tie points are shared between a couple of successive frames. This value is required in order to guarantee an appropriate level of redundancy and reproduce the actual situation: for this reason it is essential to determine the "best" number of tie points (called $\mathrm{TP})$ to be used. This value can also take into consideration the 
presence of white noise, which can be introduced with the aim of describing a more realistic situation thus obtaining convincing results (this analysis will be described in another paper).

The Authors are interested in the number of TP because a large number of TPs does not necessarily lead to better results. In fact, in a real case study, a higher number of TP could result in a higher number of outliers therefore it is essential to decide whether it is preferable to have several TP and the certainty of outliers or to "settle" for a smaller number of TP and so probably obtain fewer outliers. This decision is also influenced by the need of having the minimum number of TP required to solve the problem, which is to determine the unknown parameters, and more importantly to ensure an adequate level of redundancy.

This is a delicate issue, because the minimum number depends on the decision about the "use of the outliers" (Rousseeuw, 1987). In fact there are two trains of thought concerning this topic: the first is focused on the "re-weighting" of the outliers, the second consists in deleting the outliers and recalculating the unknown parameters with fewer TPs.

Lastly but not least the value of initial uncertainty related to the measure of the tie points was included in the simulator as another parameter of input. In the case study it is set equal to 0.15 pixel.

Regarding the type of trajectory which we have to study, the simulator enables us to set the characteristics of the object points we wish to observe.

In fact in order to simulate the trajectory of a vehicle realistically, it is important set its basic features: direction, length, distance between the centers of projection of the frames and the object points and consequentially the characteristics of the camera which we want to use, the overlap and the camera attitude.

In this way the simulator enabled us to study the effect of the various trajectories on quality: in fact by using a rectilinear rather than curvilinear trajectory, it is possible to obtain different values of error propagated for the same overlap and the same number of TPs. It is well known that it is more difficult to match the points between frames with a curvilinear trajectory than with a rectilinear trajectory due to problems concerning the overlapping of the line of flight of the horizontal elements or due to some visual occlusions which is a typical issue that occurs less frequently in the case of rectilinear trajectory where the view is generally better.

Therefore the length and type of trajectory can be established, which may be rectilinear or curvilinear and also perfectly plane or characterized by a slope. In fact the Authors decided to simulate streets with a certain longitudinal slope in order to create a real-life scenario.

Moreover, it is possible to "locate" the camera on the simulated vehicle in a specific position and establish its attitude along the trajectory and the distance between the projection centers of the frames and the object points ( $\mathrm{H}$ in Figure 2).

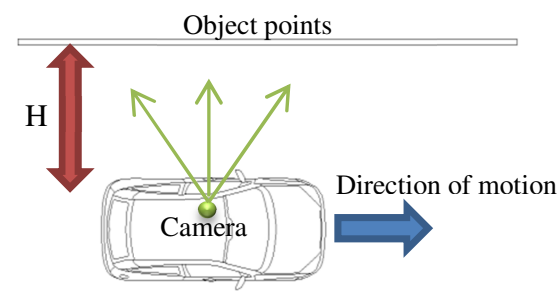

Figure 2. Schema of the simulation $(\mathrm{H}=$ object-to-camera distance)

With the aim of describing a Terrestrial Mobile Mapping relief the Authors simulated the presence of 10-meter high buildings situated quite close to the camera at a distance of 3-5 meters (the normal width of a two-lane street with pavements) in order to reproduce a typical urban canyon.

To simulate the presence of windows, windowsills, doors and balconies commonly present in a townhouse, Authors did not position the object points (which in a TMM relief obviously describes the townhouses) perfectly within the plane parallel to the direction of motion, but they shifted them by about $20 \mathrm{~cm}$ (another input value). This plane is located at the distance $\mathrm{H}$ (Figure 2 and Figure 3), which is set as input.

Moreover the trajectory is not designed perfectly parallel to the middle plane that contains the object points, but it is simulated as if it deflected by an angle introduced as input, as occurs in an urban canyon.

The arrangement of the object points is of great importance because it is related to the distribution of the TP in the overlap area. The point distribution of the buildings is realized on the object, in the terrain frame and then these points are projected into the frame system by means of collinearity equations.

The software can simulate object points in some ways: they can be distributed on a regular grid in the three directions (shown in Figure 3), they can be arranged randomly (the Authors used the quasi-random mode, shown in Figure 4) or they can be distributed in "bands", to represent the typical trend of the city buildings (Figure 5).

This first type of representation (Figure 3) is probably the best way to describe the most optimistic case study: if we realize a TMM relief, we can see that the distribution of the object points is irregular, in fact these points are generally located in parts of the overlap area.

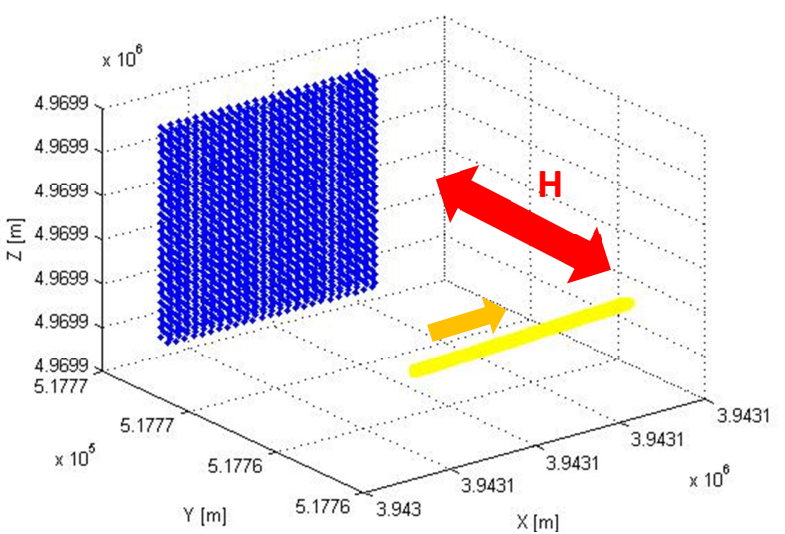

Figure 3. Blue: regular object points distribution. Red: distance between object points and the simulated trajectory (yellow) 
For this reason the Authors preferred to adopt other more realistic types of object point distribution such as the quasirandom and "band" distribution, which are described below with their results.

With the quasi-random distribution (an example is shown in Figure 4) the Authors mean the Halton distribution (Halton, 1964) in which the values are obtained according to the creation of various prime bases to then form finer uniform partitions of the unit interval in each dimension (Kocis et al, 1997 and Kuipers and Niederreiter, 2005). It is necessary to set an initial point set in order to put it into practise. The points sets are matrices of size $n-b y-d$, where $n$ is the number of points and $d$ is the dimension of integration. Standard Halton sequences perform very well in low dimensions as in this case study.

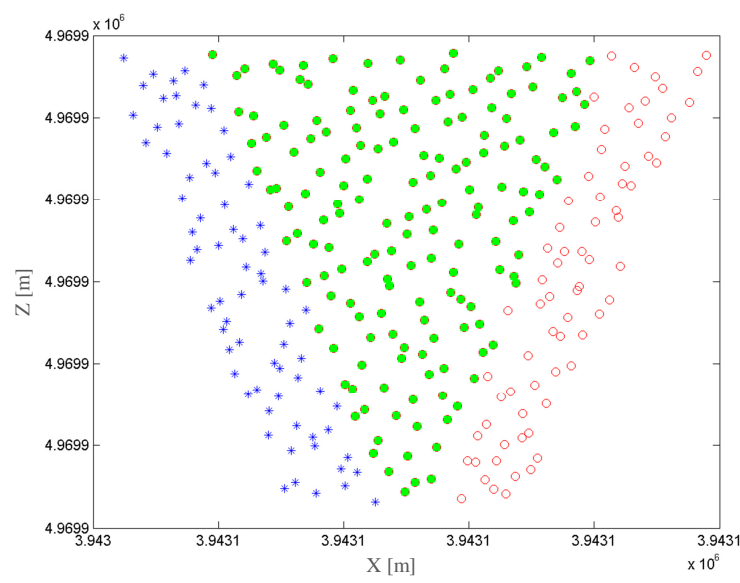

Figure 4. Example of an $80 \%$ overlap between two frames, with $150 \mathrm{TP}$ distributed in a quasi-random mode. Blue: object point of frame 1; red: object point of frame 2; green $=$ object point in the overlap area.

The third type of object point distribution is arranged in "bands" (Figure 5): it means that the Authors realized a basic module (Figure 6), which when multiplied, is used to represent the typical configuration of urban buildings, similar to an urban canyon (an example of a "sequence of simulated buildings" is visible in red in Figure 5).

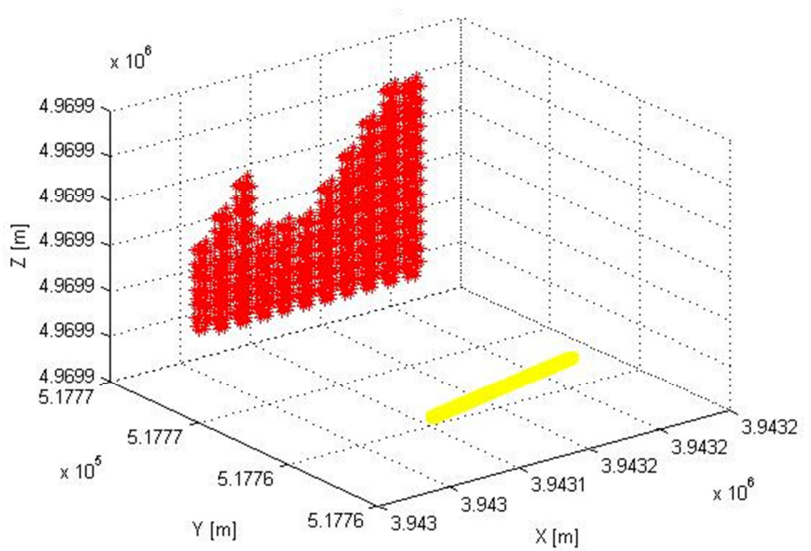

Figure 5. Red: "band" object points distribution, yellow: vehicle trajectory
This basic module is characterize by an establishing the base, height, number of doors, windows and windowsills.

The value of input base is used to realized the buildings along the trajectory: the simulator creates the maximum possible number of buildings along the trajectory characterized by the value of base alternated by its half, with the aim of modifying the pattern of the object points. Furthermore the mean value of height of the buildings and their maximum height can be set as input: so the simulator can change these height values in the limits thus modifying the geometric configuration of the buildings in the street.

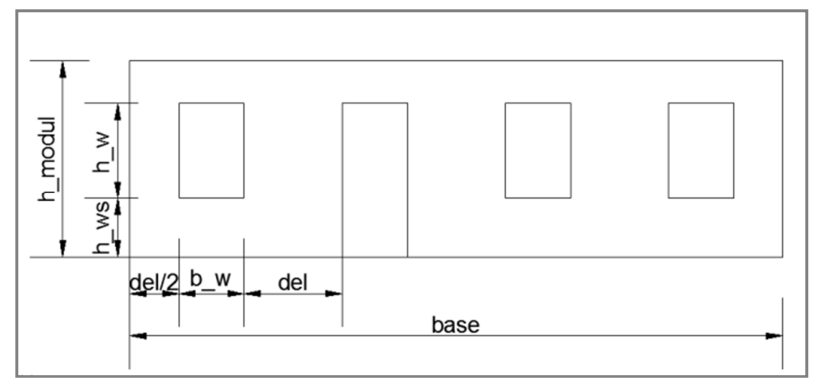

Figure 6. Basic module for the buildings and dimension value which can be introduced parametrically as input

This latest type of configuration requires a fixed number of object points in the buildings (which coincide with the corners of the modules), therefore if a greater number of points are needed, we can increase the number of points in each corner. This can be done by "creating an area of points" of fixed dimension around the corners (we can introduce the maximum distance between the corner and the additional points as input). The increase in the number of TPs for a corner of the module can be seen in Figure 7.

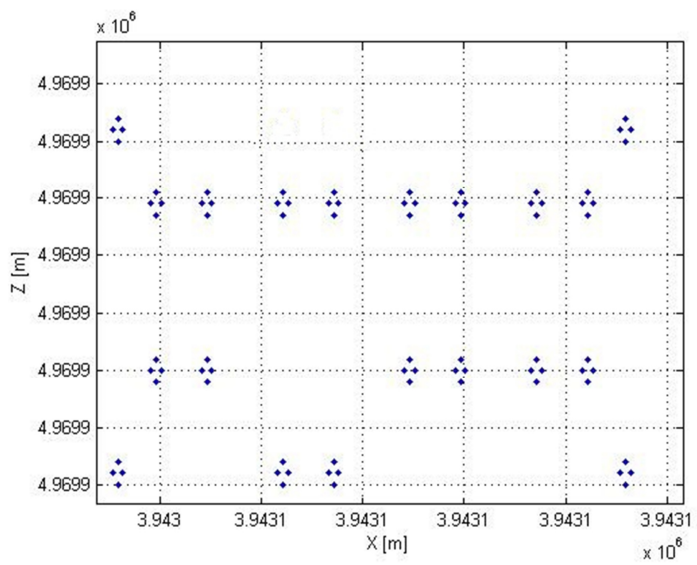

Figure 7. Example of increasing in the number of TPs for a corner of the module (4 points for each corner)

This expedient enables us to use all the overlap values and the numbers of tie points under investigation and it provides us with a clearer picture of the real situation where there is normally more than one tie point around each corner.

The Authors believe that these last two types of object point distributions (quasi-random and in "bands") are the most suitable to use because they represent a compromise between 
the ideal situation of object points placed on a regular grid and the realistic distribution of object points (a sort of random distribution). The results obtained with these last two types of configuration using a rectilinear and a curvilinear trajectory can be seen in paragraph 5 .

\section{CASE STUDY}

According to recent research trends, the Authors decided to use the parameters of the commercial low cost camera described above for the simulation, with the aim of obtaining results comparable with those obtained in a similar real case of TMM (carried out with the same type of camera and similar geometric characteristics of the relief (Angelats and Colomina, 2014 and De Agostino et al., 2010)), in order to test the reliability of the simulator.

This camera sees the "terrain points located on the left-hand side", it is placed at a certain height on the vehicle (for example at $1.50 \mathrm{~m}$ ) and moves at a established speed depending on the value of overlap desired between successive frames.

The Authors decided to analyze four level of overlap between two successive frames: $60 \%$, considered to be the minimum level of overlap required for obtaining good results (in fact it is the classical minimum value of overlap considered for aerial reliefs (Blàsquez and Colomina, 2012)); 70\%, 80\%, up to $90 \%$ of overlap.

As already mentioned in the introduction of this article, it is possible to set the length and type of trajectory, therefore a 100metre, rectilinear and curvilinear trajectory with a certain degree of declivity was considered, which was located 5 meters from and not perfectly parallel to the middle plane containing the object points (located with a quasi-random distribution and as "bands").

The next paragraph reports the results (the obtained precisions) of various case studies: in the first simulation the quasi random mode object distribution and rectilinear trajectory were used, in the second the object point distribution remained the same while the trajectory was curvilinear, the third simulation was carried out with an object point distribution made of "bands" and a rectilinear trajectory and for the last simulation a "band" object point distribution with a curvilinear trajectory were used.

The number of TP used in the cases studies are 25, 50 and 150.

The Authors did not include outliers in these analyses, as the aim of the study was to determine the maximum level of precision obtainable with photogrammetry in a TMM relief. The possible introduction of outliers, how to find a "better collocation" for them in order to make them more effective and how to identify them will be the object of future research.

As already mentioned, we cannot consider a random number of points as we have to ensure an adequate level of redundancy especially if we decide to delete some outliers instead of reweighting them. The solution will be unsatisfactory if there is a limited number of TP, e.g. less than 20 in this case study in which we have five unknown parameters The Authors wanted to determine how the accidental errors are propagated in the orientation between the frames only by means of the photogrammetric measurements so they introduced the mathematical model of asymmetric relative orientation (equation 1).This model imply the presence of five unknown parameters ( 3 of attitude and 2 of position, the scale is known) and for this reason the minimum recommended number of TP sufficient to obtain a good solution has to be 25 . Instead the maximum number of TP is chose equal to 150 which guarantees a high level of redundancy and compared to a real situation in which the extraction of one thousand points is possible, it was considered to be a precautionary, pessimistic situation. It is also analyzed an intermediate case of $50 \mathrm{TP}$.

The outputs can now be used for calculating the relative asymmetric orientation parameters between a couple of successive frames, the error propagated in the various components of attitude and position and therefore it is possible to see how precise the relief of the simulated case study actually is.

\section{MATHEMATICAL MODEL}

The mathematical model used for determining the parameters of attitude of the frames is based on the well-known asymmetric relative orientation equations (Hartley and Zisserman, 2004 and Vosselman et al., 2004).

Equation 1 developed from an implicit model, enable us to determine the unknown parameters of the problem.

$$
g\left(l, x_{i}\right)-\left(\frac{\partial g}{\partial l}\right)_{\mid\left(l, x_{i}\right)} \cdot \mathrm{v}+\left(\frac{\partial g}{\partial x}\right)_{\mid\left(l, x_{i}\right)} \cdot \Delta x_{i}=0
$$

where $\quad g=$ asymmetric relative orientation function

$\mathrm{x}=$ unknown parameters

$1=$ observations

$v=$ unknown residuals of observations

$\mathrm{D}=-\left(\frac{\partial g}{\partial l}\right)_{\mid\left(l, x_{i}\right)}=\mathrm{D}$ matrix

It is an implicit model because it consider the presence of the Dmatrix, which in this case contains the values of derivatives of the function of orientation $g$ respect to the observation $l$.

In respect to the explicit model, constructed using an easier formula $l+v=A \cdot x$, the implicit model enables us to obtain parameters in a more precise manner.

The solution thus obtained is compared to a reference trajectory known a priori.

\section{RESULTS}

The following tables show the values of error propagated $(\varepsilon)$ obtained from the various components of attitude and position, related to the overlap values and the number of TPs used.

The results shown in Table 1,2 and 3 are related to the simulation which considers a rectilinear trajectory and the quasi-random distribution for the object points with 25,50 and 150 TPs.

\begin{tabular}{|c|c|c|c|c|c|c|}
\hline $\begin{array}{c}\mathbf{N}^{\circ} \\
\mathbf{T P}\end{array}$ & $\begin{array}{c}\text { Overlap } \\
{[\mathbf{\%}]}\end{array}$ & $\begin{array}{c}\boldsymbol{\varepsilon}_{\text {ROLL }} \\
{[\mathbf{d e g}]}\end{array}$ & $\begin{array}{c}\boldsymbol{\varepsilon}_{\text {PITCH }} \\
{[\mathbf{d e g}]}\end{array}$ & $\begin{array}{c}\boldsymbol{\varepsilon}_{\text {YAW }} \\
{[\mathbf{d e g}]}\end{array}$ & $\begin{array}{c}\boldsymbol{\varepsilon}_{\text {DIR_Y }} \\
{[\mathbf{m}]}\end{array}$ & $\begin{array}{c}\boldsymbol{\varepsilon}_{\text {DIR_Z }} \\
{[\mathbf{m}]}\end{array}$ \\
\hline \multirow{3}{*}{25} & 60 & 2.42 & 10.95 & 4.91 & 0.37 & 0.77 \\
\cline { 2 - 7 } & 70 & 1.92 & 6.63 & 2.70 & 0.24 & 0.39 \\
\cline { 2 - 7 } & 80 & 1.60 & 3.31 & 1.27 & 0.18 & 0.18 \\
\cline { 2 - 7 } & 90 & 1.72 & 1.99 & 0.70 & 0.19 & 0.14 \\
\hline
\end{tabular}

Table 1. Error propagated - $25 \mathrm{TP}$ - simulation 1

\begin{tabular}{|c|c|c|c|c|c|c|}
\hline $\begin{array}{c}\mathbf{N}^{\circ} \\
\mathbf{T P}\end{array}$ & $\begin{array}{c}\text { Overlap } \\
{[\%]}\end{array}$ & $\begin{array}{c}\boldsymbol{\varepsilon}_{\text {ROLL }} \\
{[\mathbf{d e g}]}\end{array}$ & $\begin{array}{c}\boldsymbol{\varepsilon}_{\text {PITCH }} \\
{[\mathbf{d e g}]}\end{array}$ & $\begin{array}{c}\mathbf{\varepsilon}_{\text {YAW }} \\
{[\mathbf{d e g}]}\end{array}$ & $\begin{array}{c}\boldsymbol{\varepsilon}_{\text {IIR_Y }} \\
{[\mathbf{m}]}\end{array}$ & $\begin{array}{c}\boldsymbol{\varepsilon}_{\text {DIR_Z }} \\
{[\mathbf{m}]}\end{array}$ \\
\hline \multirow{2}{*}{$\mathbf{5 0}$} & 60 & 1.57 & 6.81 & 3.02 & 0.24 & 0.46 \\
\cline { 2 - 7 } & 70 & 1.26 & 4.18 & 1.73 & 0.16 & 0.25 \\
\hline
\end{tabular}




\begin{tabular}{|l|l|l|l|l|l|l|} 
& 80 & 1.10 & 2.20 & 0.85 & 0.12 & 0.12 \\
\cline { 2 - 7 } & 90 & 1.07 & 1.27 & 0.46 & 0.12 & 0.09 \\
\hline
\end{tabular}

Table 2. Error propagated $-50 \mathrm{TP}-$ simulation 1

\begin{tabular}{|c|c|c|c|c|c|c|}
\hline $\begin{array}{c}\mathbf{N}^{\circ} \\
\mathbf{T P}\end{array}$ & $\begin{array}{c}\text { Overlap } \\
{[\%]}\end{array}$ & $\begin{array}{c}\boldsymbol{\varepsilon}_{\text {ROLL }} \\
{[\mathbf{d e g}]}\end{array}$ & $\begin{array}{c}\boldsymbol{\varepsilon}_{\text {PITCH }} \\
{[\mathbf{d e g}]}\end{array}$ & $\begin{array}{c}\boldsymbol{\varepsilon}_{\text {YAW }} \\
{[\mathbf{d e g}]}\end{array}$ & $\begin{array}{c}\boldsymbol{\varepsilon}_{\text {DIR_Y }} \\
{[\mathbf{m}]}\end{array}$ & $\begin{array}{c}\boldsymbol{\varepsilon}_{\text {DIR_Z }} \\
{[\mathbf{m}]}\end{array}$ \\
\hline \multirow{3}{*}{$\mathbf{1 5 0}$} & 60 & 0.95 & 4.08 & 1.81 & 0.14 & 0.27 \\
\cline { 2 - 7 } & 70 & 0.67 & 2.45 & 1.02 & 0.09 & 0.14 \\
\cline { 2 - 7 } & 80 & 0.60 & 1.21 & 0.47 & 0.07 & 0.07 \\
\cline { 2 - 8 } & 90 & 0.57 & 0.72 & 0.27 & 0.07 & 0.05 \\
\hline
\end{tabular}

Table 3. Error propagated - $150 \mathrm{TP}$ - simulation 1

The second simulation refers to a quasi-random distribution of the object points and a curvilinear trajectory (Figure 8 which has the same characteristics as the rectilinear trajectory described above.

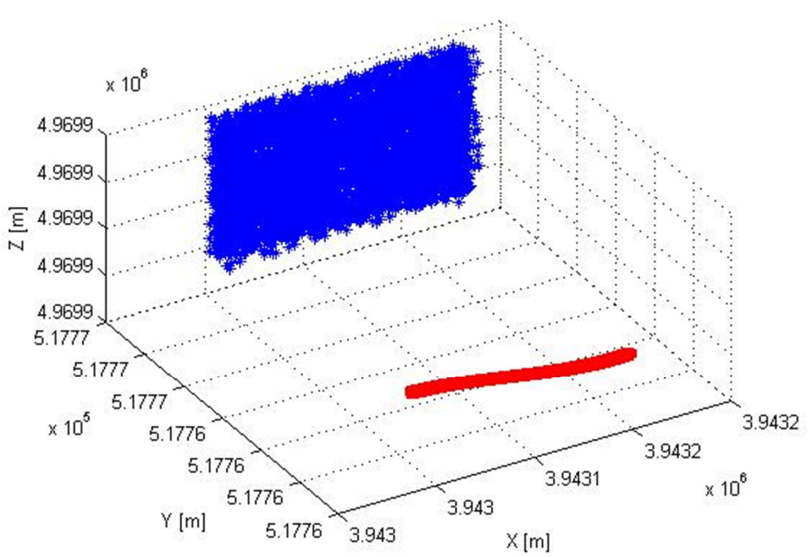

Figure 8. Example of quasi-random object points distribution (blue) and curvilinear trajectory (red)

Table 4, 5 and 6 shows the results related to the second case study: quasi-random object points distribution and curvilinear trajectory (shown in Figure 8).

\begin{tabular}{|c|c|c|c|c|c|c|}
\hline $\begin{array}{c}\mathbf{N}^{\circ} \\
\mathbf{T P}\end{array}$ & $\begin{array}{c}\text { Overlap } \\
{[\%]}\end{array}$ & $\begin{array}{c}\boldsymbol{\varepsilon}_{\text {ROLL }} \\
{[\mathbf{d e g}]}\end{array}$ & $\begin{array}{c}\boldsymbol{\varepsilon}_{\text {PITCH }} \\
{[\mathbf{d e g}]}\end{array}$ & $\begin{array}{c}\boldsymbol{\varepsilon}_{\text {YAW }} \\
{[\mathbf{d e g}]}\end{array}$ & $\begin{array}{c}\boldsymbol{\varepsilon}_{\text {DIR_Y }} \\
{[\mathbf{m}]}\end{array}$ & $\begin{array}{c}\boldsymbol{\varepsilon}_{\text {DIR_Z }} \\
{[\mathbf{m}]}\end{array}$ \\
\hline \multirow{3}{*}{25} & 60 & 5.18 & 22.94 & 10.10 & 0.74 & 1.57 \\
\cline { 2 - 7 } & 70 & 2.82 & 9.76 & 3.93 & 0.35 & 0.56 \\
\cline { 2 - 7 } & 80 & 2.61 & 4.62 & 1.34 & 0.29 & 0.26 \\
\cline { 2 - 7 } & 90 & 2.58 & 2.90 & 0.90 & 0.29 & 0.20 \\
\hline
\end{tabular}

Table 4. Error propagated - $25 \mathrm{TP}$ - simulation 2

\begin{tabular}{|c|c|c|c|c|c|c|}
\hline $\begin{array}{c}\mathbf{N}^{\circ} \\
\mathbf{T P}\end{array}$ & $\begin{array}{c}\text { Overlap } \\
{[\mathbf{\%}]}\end{array}$ & $\begin{array}{c}\boldsymbol{\varepsilon}_{\text {ROLL }} \\
{[\mathbf{d e g}]}\end{array}$ & $\begin{array}{c}\boldsymbol{\varepsilon}_{\text {PITCH }} \\
{[\mathbf{d e g}]}\end{array}$ & $\begin{array}{c}\boldsymbol{\varepsilon}_{\text {YAW }} \\
{[\mathbf{d e g}]}\end{array}$ & $\begin{array}{c}\boldsymbol{\varepsilon}_{\text {DIR_Y }} \\
{[\mathbf{m}]}\end{array}$ & $\begin{array}{c}\boldsymbol{\varepsilon}_{\text {DIR_Z }} \\
{[\mathbf{m}]}\end{array}$ \\
\hline \multirow{3}{*}{$\mathbf{5} 5$} & 60 & 2.70 & 13.02 & 5.54 & 0.42 & 0.87 \\
\cline { 2 - 7 } & 70 & 1.93 & 6.93 & 2.74 & 0.25 & 0.38 \\
\cline { 2 - 7 } & 80 & 1.72 & 3.23 & 1.14 & 0.19 & 0.17 \\
\cline { 2 - 7 } & 90 & 1.59 & 1.80 & 0.61 & 0.18 & 0.22 \\
\hline
\end{tabular}

Table 5. Error propagated - $50 \mathrm{TP}$ - simulation 2

\begin{tabular}{|c|c|c|c|c|c|c|}
\hline $\begin{array}{c}\mathbf{N}^{\circ} \\
\mathbf{T P}\end{array}$ & $\begin{array}{c}\text { Overlap } \\
{[\%]}\end{array}$ & $\begin{array}{c}\boldsymbol{\varepsilon}_{\text {ROLL }} \\
{[\mathbf{d e g}]}\end{array}$ & $\begin{array}{c}\boldsymbol{\varepsilon}_{\text {PITCH }} \\
{[\mathbf{d e g}]}\end{array}$ & $\begin{array}{c}\boldsymbol{\varepsilon}_{\text {YAW }} \\
{[\mathbf{d e g}]}\end{array}$ & $\begin{array}{c}\boldsymbol{\varepsilon}_{\text {DIR_Y }} \\
{[\mathbf{m}]}\end{array}$ & $\begin{array}{c}\boldsymbol{\varepsilon}_{\text {DIR_Z }} \\
{[\mathbf{m}]}\end{array}$ \\
\hline \multirow{3}{*}{$\mathbf{1 5 0}$} & 60 & 1.57 & 7.96 & 3.43 & 0.26 & 0.52 \\
\cline { 2 - 8 } & 70 & 1.03 & 3.86 & 1.54 & 0.14 & 0.22 \\
\cline { 2 - 8 } & 80 & 0.96 & 1.84 & 0.66 & 0.11 & 0.09 \\
\cline { 2 - 8 } & 90 & 0.90 & 1.02 & 0.35 & 0.10 & 0.06 \\
\hline
\end{tabular}

Table 6. Error propagated - $150 \mathrm{TP}$ - simulation 2

Related to the latest two simulation, the Authors decided to show the results obtained with 80 and $90 \%$ of overlap, because for the specific object points distribution adopted (Figure 5), values of overlap lesser than 80 and $90 \%$ do not produce acceptable results.

In the following tables (Table 7, 8 and 9) there are the results obtained used the "bands" distribution and the rectilinear trajectory.

\begin{tabular}{|c|c|c|c|c|c|c|}
\hline $\begin{array}{c}\mathbf{N}^{\circ} \\
\mathbf{T P}\end{array}$ & $\begin{array}{c}\text { Overlap } \\
{[\%]}\end{array}$ & $\begin{array}{c}\boldsymbol{\varepsilon}_{\text {ROLL }} \\
{[\mathbf{d e g}]}\end{array}$ & $\begin{array}{c}\boldsymbol{\varepsilon}_{\text {PITCH }} \\
{[\mathbf{d e g}]}\end{array}$ & $\begin{array}{c}\mathbf{\varepsilon}_{\text {YAW }} \\
{[\mathbf{d e g}]}\end{array}$ & $\begin{array}{c}\boldsymbol{\varepsilon}_{\text {DIR_Y }} \\
{[\mathbf{m}]}\end{array}$ & $\begin{array}{c}\boldsymbol{\varepsilon}_{\text {DIR_Z }} \\
{[\mathbf{m}]}\end{array}$ \\
\hline \multirow{2}{*}{25} & 80 & 1.67 & 3.82 & 0.95 & 0.17 & 0.22 \\
\cline { 2 - 7 } & 90 & 2.63 & 2.92 & 0.84 & 0.27 & 0.20 \\
\hline
\end{tabular}

Table 7. Error propagated - $25 \mathrm{TP}$ - simulation 3

\begin{tabular}{|c|c|c|c|c|c|c|}
\hline $\begin{array}{c}\mathbf{N}^{\circ} \\
\mathbf{T P}\end{array}$ & $\begin{array}{c}\text { Overlap } \\
{[\%]}\end{array}$ & $\begin{array}{c}\boldsymbol{\varepsilon}_{\text {ROLL }} \\
{[\mathbf{d e g}]}\end{array}$ & $\begin{array}{c}\boldsymbol{\varepsilon}_{\text {PITCH }} \\
{[\mathbf{d e g}]}\end{array}$ & $\begin{array}{c}\mathbf{\varepsilon}_{\text {YAW }} \\
{[\mathbf{d e g}]}\end{array}$ & $\begin{array}{c}\boldsymbol{\varepsilon}_{\text {DIR_Y }} \\
{[\mathbf{m}]}\end{array}$ & $\begin{array}{c}\boldsymbol{\varepsilon}_{\text {DIR_Z }} \\
{[\mathbf{m}]}\end{array}$ \\
\hline \multirow{2}{*}{$\mathbf{5 0}$} & 80 & 1.40 & 2.55 & 0.74 & 0.14 & 0.16 \\
\cline { 2 - 7 } & 90 & 1.51 & 1.63 & 0.50 & 0.16 & 0.13 \\
\hline
\end{tabular}

Table 8. Error propagated - $50 \mathrm{TP}$ - simulation 3

\begin{tabular}{|c|c|c|c|c|c|c|}
\hline $\begin{array}{c}\mathbf{N}^{\circ} \\
\mathbf{T P}\end{array}$ & $\begin{array}{c}\text { Overlap } \\
{[\%]}\end{array}$ & $\begin{array}{c}\boldsymbol{\varepsilon}_{\text {ROLL }} \\
{[\mathbf{d e g}]}\end{array}$ & $\begin{array}{c}\boldsymbol{\varepsilon}_{\text {PITCH }} \\
{[\mathbf{d e g}]}\end{array}$ & $\begin{array}{c}\boldsymbol{\varepsilon}_{\text {YAW }} \\
{[\mathbf{d e g}]}\end{array}$ & $\begin{array}{c}\boldsymbol{\varepsilon}_{\text {DIR_Y }} \\
{[\mathbf{m}]}\end{array}$ & $\begin{array}{c}\boldsymbol{\varepsilon}_{\text {DIR_Z }} \\
{[\mathbf{m}]}\end{array}$ \\
\hline \multirow{2}{*}{$\mathbf{1 5 0}$} & 80 & 0.93 & 1.59 & 0.49 & 0.09 & 0.10 \\
\cline { 2 - 7 } & 90 & 0.90 & 0.96 & 0.31 & 0.09 & 0.08 \\
\hline
\end{tabular}

Table 9. Error propagated - $150 \mathrm{TP}$ - simulation 3

Latest tables (Table 10, 11 and 12), show the results related to "bands" distribution for the object points and the curvilinear trajectory.

\begin{tabular}{|c|c|c|c|c|c|c|}
\hline $\begin{array}{c}\mathbf{N}^{\circ} \\
\mathbf{T P}\end{array}$ & $\begin{array}{c}\text { Overlap } \\
{[\%]}\end{array}$ & $\begin{array}{c}\boldsymbol{\varepsilon}_{\text {ROLL }} \\
{[\mathbf{d e g}]}\end{array}$ & $\begin{array}{c}\boldsymbol{\varepsilon}_{\text {PITCH }} \\
{[\mathbf{d e g}]}\end{array}$ & $\begin{array}{c}\boldsymbol{\varepsilon}_{\text {YAW }} \\
{[\mathbf{d e g}]}\end{array}$ & $\begin{array}{c}\boldsymbol{\varepsilon}_{\text {DIR_Y }} \\
{[\mathbf{m}]}\end{array}$ & $\begin{array}{c}\boldsymbol{\varepsilon}_{\text {DIR_Z }} \\
{[\mathbf{m}]}\end{array}$ \\
\hline \multirow{2}{*}{$\mathbf{2 5}$} & 80 & 3.12 & 5.12 & 1.93 & 0.32 & 0.27 \\
\cline { 2 - 7 } & 90 & 3.88 & 3.24 & 0.85 & 0.39 & 0.27 \\
\hline
\end{tabular}

Table 10. Error propagated - $25 \mathrm{TP}$ - simulation 4

\begin{tabular}{|c|c|c|c|c|c|c|}
\hline $\begin{array}{c}\mathbf{N}^{\circ} \\
\mathbf{T P}\end{array}$ & $\begin{array}{c}\text { Overlap } \\
{[\%]}\end{array}$ & $\begin{array}{c}\boldsymbol{\varepsilon}_{\text {ROLL }} \\
{[\mathbf{d e g}]}\end{array}$ & $\begin{array}{c}\boldsymbol{\varepsilon}_{\text {PITCH }} \\
{[\mathbf{d e g}]}\end{array}$ & $\begin{array}{c}\boldsymbol{\varepsilon}_{\text {YAW }} \\
{[\mathbf{d e g}]}\end{array}$ & $\begin{array}{c}\boldsymbol{\varepsilon}_{\text {DIR_Y }} \\
{[\mathbf{m}]}\end{array}$ & $\begin{array}{c}\boldsymbol{\varepsilon}_{\text {DIR_Z }} \\
{[\mathbf{m}]}\end{array}$ \\
\hline $\mathbf{5 0}$ & 80 & 2.55 & 3.76 & 1.18 & 0.26 & 0.22 \\
\hline
\end{tabular}




\begin{tabular}{|l|l|l|l|l|l|l|}
\hline & 90 & 2.41 & 2.17 & 0.61 & 0.25 & 0.17 \\
\hline
\end{tabular}

Table 11. Error propagated - $50 \mathrm{TP}$ - simulation 4

\begin{tabular}{|c|c|c|c|c|c|c|}
\hline $\begin{array}{c}\mathbf{N}^{\circ} \\
\mathbf{T P}\end{array}$ & $\begin{array}{c}\text { Overlap } \\
{[\%]}\end{array}$ & $\begin{array}{c}\boldsymbol{\varepsilon}_{\text {ROLL }} \\
{[\mathbf{d e g}]}\end{array}$ & $\begin{array}{c}\boldsymbol{\varepsilon}_{\text {PITCH }} \\
{[\mathbf{d e g}]}\end{array}$ & $\begin{array}{c}\boldsymbol{\varepsilon}_{\text {YAW }} \\
{[\mathbf{d e g}]}\end{array}$ & $\begin{array}{c}\boldsymbol{\varepsilon}_{\text {DIR_Y }} \\
{[\mathbf{m}]}\end{array}$ & $\begin{array}{c}\boldsymbol{\varepsilon}_{\text {DIR_Z }} \\
{[\mathbf{m}]}\end{array}$ \\
\hline \multirow{2}{*}{$\mathbf{1 5 0}$} & 80 & 1.58 & 2.36 & 0.67 & 0.16 & 0.14 \\
\cline { 2 - 7 } & 90 & 1.70 & 1.55 & 0.42 & 0.17 & 0.11 \\
\hline
\end{tabular}

Table 12. Error propagated - $150 \mathrm{TP}$ - simulation 4

The results obtained from the simulations show an increase in the error propagated for all components if a smaller overlap and fewer TPs are considered. As already mentioned, the results are of better quality if we use a rectilinear trajectory rather than a curvilinear trajectory due to matching or occlusion issues as you can see in the previous tables.

If we consider the "bands" object points distribution it is recommendable to adopt values of overlap like $80 \%$, because the specific distribution of these determines unacceptable results for lesser values of overlap.

Therefore good results were obtained with the simulator as they were similar to a real case realized with the same camera and had the same "street configuration characteristics. As in an real case, if the value of overlap between the couple of images increases, there is a decrease in the error propagated in the various angular components and in the components of position of relative orientation in the sequence of images.

The worst result of the angular component is pitch, it can be justified by the specific geometric configuration of the case study and it is related to the specific collocation of the camera type.

When the value of overlap increased, a reduction of the error propagated related to all the components in the study was observed, and this trend can also be seen for the angular component pitch. This trend is justifiable by the increase in the base distance ratio. Figure 9 shows an example of this: in the first image on the left we can see an overlap of $60 \%$, where the base (B) is smaller than in the second image in which the overlap is $90 \%$.

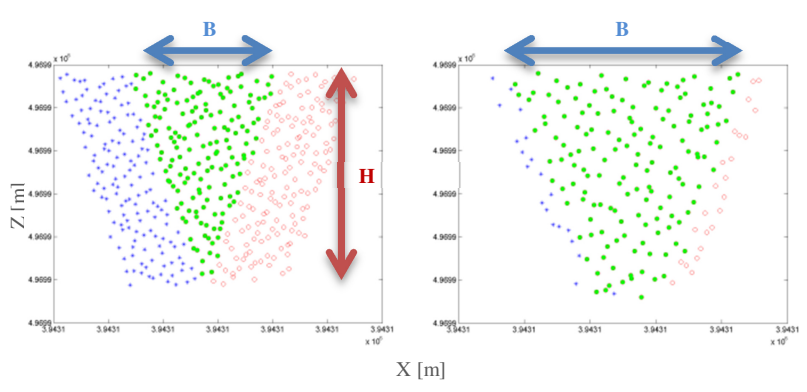

Figure 9. On the left side: $60 \%$ of overlap and $150 \mathrm{TP}$, on the right side: $90 \%$ of overlap and $150 \mathrm{TP}$

\section{CONCLUSION}

By observing the results it is clear that the simulator works effectively.

This simulator enables us to design a TMM relief in office, before going in the field and it prevents us from creating an incorrect relief. It also helps us to predict the value of the obtainable precision and modify the parameters of the camera in order to obtain the level of precision required for the specific case under examination.
The possibility of carrying out tests similar relief by modifying the configuration of the instruments saves time and money, as we are able to solve the various problems and add devices and improvements to the methodology used for realizing the relief. The simulator enables us to test the place where we must carry out the TMM relief and identify the best configuration for the instruments at our disposal, the most suitable type of equipment (camera type and the minimum number of cameras to be used) and it enables us to identify the minimum value of overlap between successive frames required for obtaining a good result, with the correct value of precision required with photogrammetric information (De Agostino et al., 2009).

In conclusion the Authors believe that photogrammetry is a useful tool for positioning when there is no GPS signal as occurs in an urban canyon, and their goal is to develop the best design project for obtaining a good photogrammetric result for the specific relief in use.

The results obtained following this approach can later be fed into a more complex simulator which takes other types of sensors into consideration (De Agostino et al, 2011) and thus design the obtainable level of precision of a TMM relief when there is no GNSS signal.

\section{ACKNOWLEDGEMENTS}

The Authors would like to thank the Director of the Geomatics Division of the CTTC (Centre Tecnològic de Telecomunicacions de Catalunya) Castelldefels (Spain), Dr. Ismael Colomina and his staff the support.

\section{REFERENCES}

Angelats E., Colomina I., 2014. One step mobile mapping laser and camera data orientation and calibration. ISPRS Archives, Vol. XL-3/W1

Blàsquez M., Colomina I., 2012. Relative INS/GNSS aerial control in integrated sensor orientation: Models and performance. ISPRS Journal of Photogrammetry and Remote Sensing, Vol.67, pp. 120-133

De Agostino M., Lingua A., Marenchino D., Nex F., Piras M., 2011. GIMPHI: a new integration approach for early impact assessment. Applied Geomatics, Vol. 3(4), pp. 241-249

De Agostino M., Manzino A.M., Piras M., 2010. Performance comparison of different MEMS-based IMUs. Position Location and Navigation Symposium (PLANS), IEEE/ION, pp. 87-201

De Agostino M., Lingua A., Marenchino D., Nex F., Piras M., 2009. A new photogrammetric combined approach to improve the GNSS/INS solution. ION GNSS 2009, Savannah (USA GA), 22-25 September 2009. pp. 460-470

El-Sheimy N., 1996. A Mobile Multi-Sensor System for GIS Applications in Urban Centers. International Archives of Photogrammetry and Remote Sensing, Vol. XXXI, pp. 95-100

Halton J., 1964. Algorithm 247: Radical-inverse quasi-random point sequence. Communications of the ACM, Vol. 7 issue 12, pp.701-702

Hartley, R., Zisserman, A., 2004. Multiple vie geometry in Computer Vision. Cambridge University Press

Hassan T., Ellum C., El-Sheimy N., 2007. Bridging land-based mobile mapping using photogrammetric adjustments. ISPRS 
Commission Technique I. Symposium, Marne-la-Vallée, France, Vol. 185 , pp. 67-72

Kocis L., Whiten W.J., 1997. Computational Investigations of Low-Discrepancy Sequences. ACM Transactions on Mathematical Software. Vol. 23 (2), pp. 266-294

Kuipers, L., Niederreiter H., 2005. Uniform distribution of sequences. Dover Publications, pp. 129

Rousseeuw P., Leroy A.M., 1987. Robust regression and outliers detection. Wiley series in probability and mathematical statistics. John Wiley \& Sons

Vosselman G., Sester M., Mayer, H., 2004. Basic computer vision techniques. In: McGlone, J.C., Mikhail, E.M, Bethel, J., Mullen, R. (Eds.), Manual of Photogrammetry, fifth ed. American Society of Photogrammetry and Remote Sensing, Bethesda, MA, pp. 455-504 